\title{
Evaluation of Bulk and Surface Radiation Damage of Silicon Sensors for the ATLAS Upgrade
}

\author{
Marcela Mikeštíková ${ }^{* \dagger}$, Zdeněk Kotek, Jan Št’astný \\ Institute of Physics, AS CR, Prague, Czech Republic \\ E-mail: marcela.mikestikova@cern.ch
}

\begin{abstract}
The electrical characteristics of different types of end-cap miniature $n^{+}$-in- $p$ strip sensors, ATLAS12A, were evaluated in Institute of Physics in Prague before and after proton and gamma irradiation. We report here on the bulk damage aspects, including the increase of leakage current and evaluation of the full depletion voltage and the surface damage, including the decrease of inter-strip resistance, changes in inter-strip capacitance and the effectiveness of punch-through protection structure. It was verified that different geometries of end-cap sensors do not influence their stability; the sensors should provide acceptable strip isolation and new gate PTP structure functions well even at the highest tested proton fluence $2 \times 10^{15} \mathrm{n}_{\mathrm{eq}} / \mathrm{cm}^{2}$.
\end{abstract}

The 23rd International Workshop on Vertex Detectors

15-19 September 2014

Macha Lake, The Czech Republic

\footnotetext{
*Speaker

${ }^{\dagger}$ for the ATLAS Upgrade Strip Sensor Collaboration: University of Birmingham, BNL, Cambridge University, DESY, University of Freiburg, University of Geneva, Glasgow university, KEK, Kyoto University of Education, Lancaster University, University of Liverpool, JSI and University of Ljubljana, University of New Mexico, NIKHEF, Osaka University, Charles University in Prague, Academy of Sciences of Czech R., Queen Mary University of London, UC Santa Cruz, University of Sheffield, Tokyo IT, University of Tsukuba, IFIC Valencia, and HPK
} 


\section{Introduction}

The upgrade of the Large Hadron Collider (LHC) to the higher luminosity $\left(5 \times 10^{34} \mathrm{~cm}^{-2} \mathrm{~s}^{-1}\right)$ machine, called the High Luminosity-LHC (HL-LHC), will require replacement of the entire current ATLAS Inner Detector with a new type and design of silicon sensors: pixel sensors and micro-strip sensors.

Silicon micro-strip sensors in the upgraded ATLAS experiment at the HL-LHC will be exposed to particle fluences of up to $1.2 \times 10^{15} \mathrm{n}_{\mathrm{eq}} / \mathrm{cm}^{2}$ and ionizing doses of up to $500 \mathrm{kGy}$ [1] which will substantively affect their properties: bulk damage will increase the leakage current (I) and the voltage needed for full depletion $\left(\mathrm{V}_{\mathrm{FD}}\right)$, while surface damage will raise the charge at the $\mathrm{SiO}_{2}-\mathrm{Si}$ interface that will in turn alter the inter-strip properties.

The current ATLAS Inner Detector is based on micro-strip sensors made with $p$-strips implanted on $n$-type silicon bulk ( $p$-in- $n$ ). Radiation harder $n^{+}$-in- $p$ type sensors are being considered for use in the HL-LHC trackers. This type of sensor collects electrons and has no radiation induced type inversion; it always depletes from the segmented side and thus it allows sensor operation in partially depleted mode after high fluencies when the full depletion voltage becomes high. $n^{+}$-in- $p$ type sensor is produced in a single side process that is $30-40 \%$ cheaper than the double side $n$-in- $n$ process. However, $n^{+}$-in- $p$ sensors need additional strip isolation.

A large area $n^{+}$-in- $p$ silicon sensor has been developed by the ATLAS Upgrade Strip collaboration $[2,3]$ and produced by Hamamatsu Photonics (HPK) [4] in a 6-inch (150 mm) wafer. Besides the large sensor $(9.75 \mathrm{~cm} \times 9.75 \mathrm{~cm})$, the wafer also includes sets of miniature sensors, which are used for irradiation studies. The sensors are irradiated by different particles, energies and doses and are distributed for extensive test to many institutions of the Strip collaboration".

The samples for the irradiation study presented in this paper are ATLAS12A end-cap (EC) miniature strip sensors with strips in a fan geometry. The isolation is done with common $p$ implant ( $p$-stop). The sensors vary in strip pitch, in stray strip ganging, in fan geometry (stereo strips or skewed layout) or in "punch-through protection" structure (PTP) [3]. The samples have been irradiated with protons at Birmingham [5] and Karlsruhe [6] up to fluences of $5 \times 10^{14}$, $1 \times 10^{15}$ and $2 \times 10^{15} \mathrm{n}_{\mathrm{eq}} / \mathrm{cm}^{2}$ and with gamma in BNL [7] to doses of 10,30 and $100 \mathrm{kGy}$. We report here on the bulk damage aspects, including the increase of leakage current and evaluation of the full depletion voltage as well as the surface damage, including the decrease of inter-strip resistance, changes in inter-strip capacitance and in the effectiveness of punch-through protection structure.

Previous studies performed at the Institute of Physics, Prague were done using nonirradiated large area ATLAS07 sensors [8] and neutron irradiated ATLAS07 barrel miniature sensors [9].

\section{Samples and irradiation}

The micro-strip silicon sensors studied in this work are ATLAS12A end-cap miniature sensors fabricated in $p$-type, float zone wafers with $<100>$ crystal orientation and thickness of $320 \mu \mathrm{m}$. The sensors have capacitively coupled readout $n$-strips biased through polysilicon resistors. The ATLAS12 strip sensor design [3], which was developed from the ATLAS07 
design [2], now includes miniature prototypes of the end-cap sensors with fan geometry of strips.
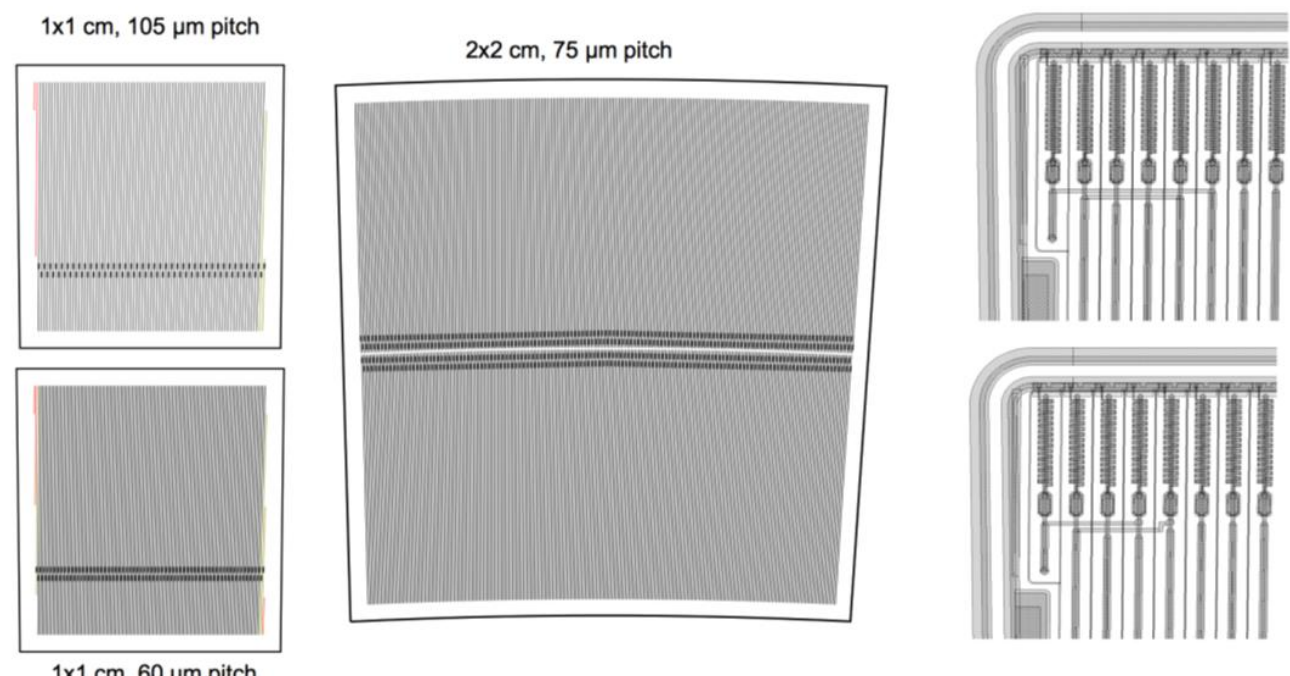

Figure 1: End-cap miniature sensors with fan geometry of strips: with stereo strips $(\sim 1 \times 1 \mathrm{~cm})$ of large pitch (upper left) or of small strip pitch (lower left) and with "skewed" layout $(\sim 2 \times 2 \mathrm{~cm})$ with two segments of strips (middle). End-cap miniature sensors with two types of stray strips ganging (AC ganging on upper right figure and DC ganging on lower right figure)

There are two types of end-cap miniature sensor geometries: $\sim 1 \times 1 \mathrm{~cm}^{2}$ with a symmetrical trapezoid geometry and $\sim 2 \times 2 \mathrm{~cm}^{2}$ with a "skewed" trapezoid geometry (Fig. 1). In both cases the strips in fan geometry are inclined at an angle of $20 \mathrm{mrad}$ to the axis of a sensor. The stereo angle of $40 \mathrm{mrad}$ is achieved with a pair of stereo sensors. There are two variations of the $1 \times 1 \mathrm{~cm}$ sensors with strips of large and small pitches: 103.4 and $64.3 \mu \mathrm{m}$ on average, respectively. The first and last two strips of the symmetrical geometry type are shorter, "blind", strips without any direct connection to readout electronics. The connection of those stray strips to normal sized strips is made by so called AC-ganging (connection to the AC readout strip metals) or DC-ganging (connection between strip implants) (Fig. 1). The skewed sensors have edges parallel to the strips and thus there are no stray strips. More details of the design including strip isolation structure are described in Ref. [3].

Strip sensors in the Inner Tracker will be exposed to charged particles, neutrons and gammas. The predicted maximum fluence and ionizing dose [1] for the integrated luminosity of $3000 \mathrm{fb}^{-1}$ in the strip end-caps are $8.1 \times 10^{14} \mathrm{~cm}^{-2}$ and $288 \mathrm{kGy}$ in the inner regions of the outermost disk at $\mathrm{z}=300 \mathrm{~cm}$ (no safety factors have been applied). Such a high fluence of particles and ionizing dose causes bulk and surface damage to the sensors. In this study the endcap miniature sensors were irradiated by protons and gammas from ${ }^{60} \mathrm{Co}$ source. Protons displace silicon atoms via non-ionizing energy losses, which results in bulk damage and ionizes both the $\mathrm{Si}$ atoms and the molecules in the insulating layer $\left(\mathrm{SiO}_{2}\right)$. The latter causes the build-up of positive charges and traps in the $\mathrm{SiO}_{2}$ and at the $\mathrm{Si}_{-} \mathrm{SiO}_{2}$ interface that leads to damage at the sensor surface. The gamma ray is usually used to isolate the oxide/interface damage from the bulk damage. However, gamma rays from ${ }^{60} \mathrm{Co}$ source are absorbed in the sensor mainly through Compton scattering and the scattered Compton electrons have high enough energy (hundreds of $\mathrm{keV}$ ) to produce point defects in the Si bulk.

The proton irradiations were performed at KIT [6] with $23 \mathrm{MeV}$ protons and at Birmingham [5] with $27 \mathrm{MeV}$ protons. Samples were kept in a cooled box during irradiation. 
The box was scanned during irradiation to cover the whole target area. The $10^{15} \mathrm{n}_{\mathrm{eq}} / \mathrm{cm}^{2}$ fluence took 15 minutes at KIT and $\sim 80 \mathrm{~s}$ in the Birmingham cyclotron. The irradiated samples were immediately stored in a refrigerator to prevent any post-irradiated annealing taking place. ${ }^{60} \mathrm{Co}$ gamma irradiation was carried out at the Solid State Gamma-ray irradiation facility of the Brookhaven National Laboratory [7]. The sensors were maintened at room temperature during irradiation. The dose rate was $220 \mathrm{~Gy} /$ hour and the $100 \mathrm{kGy}$ dose thus took 19 days in total. The dose rate uncertainty is $10 \%$.

\section{Experimental methods and results}

The sensors have been measured at the silicon laboratory at the Institute of Physics in Prague on a KarlSuss PA200 semi-automatic probe station (Fig. 2a), and on a manual probe station with a cooled chuck. Both gamma irradiated and non-irradiated sensors have been tested at room temperature. The proton irradiated sensors were tested at $-10^{\circ} \mathrm{C}$ with cool nitrogen flow. The cooling of probe station chuck was performed by a Peltier module (Fig. 2b).
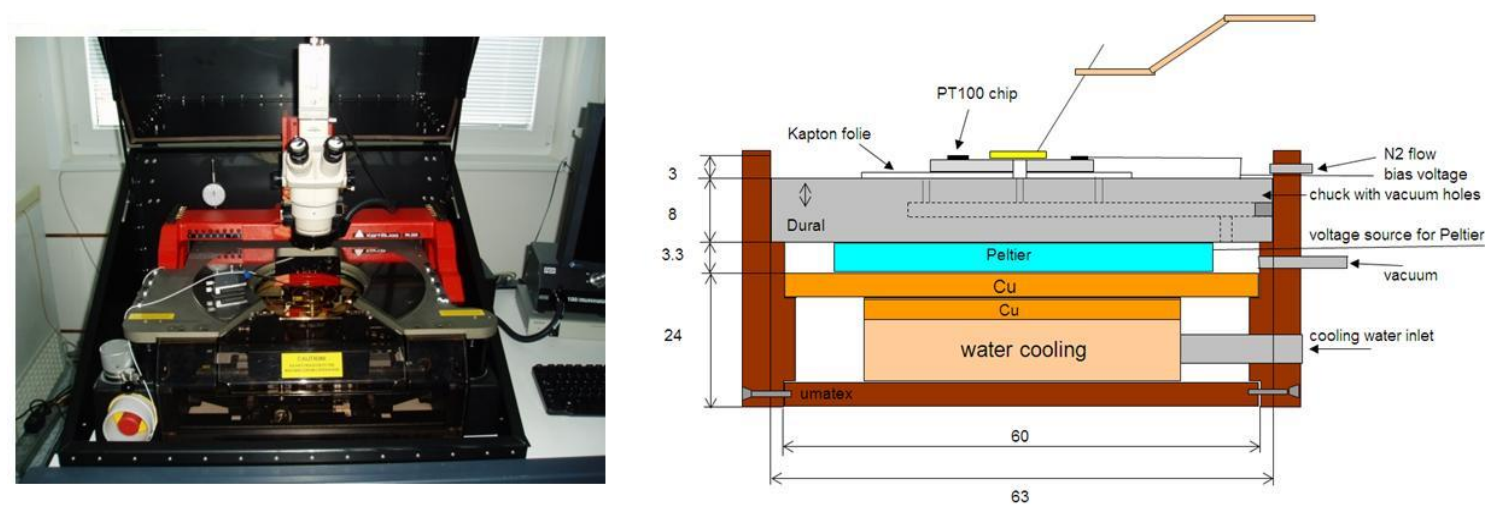

Figure 2: a) Semi-automatic probe station KarlSuss PA200 in Prague (left), b) Peltier module used for cooling the manual probe station chuck (right).

\subsection{Bulk damage}

\subsubsection{Leakage current}

The leakage current as a function of bias voltage (IV) for all the tested end-cap miniature sensors before and after proton and gamma irradiation is shown in Fig. 3. Current was measured with a delay of $10 \mathrm{~s}$ after setting the reverse bias voltage.

For non-irradiated sensors, the IV characteristics have been measured up to $600 \mathrm{~V} / 1000 \mathrm{~V}$. There were no observed onsets of micro-discharges in any of them. It shows that different geometries of end-cap sensors do not influence their stability. For all the tested non-irradiated sensors, the value of the leakage current at $600 \mathrm{~V}$ is less than $4 \mathrm{nA} / \mathrm{cm}^{2}$ which is well below the technical specification's limit $\left(<2 \mu \mathrm{A} / \mathrm{cm}^{2}\right)$ [10]. The small difference in the leakage current value of sensors with small and large pitch, as seen in Fig.3, is due to small differences in the active area of these two types of sensors. No micro-discharge breakdown was observed up to $1000 \mathrm{~V}$ sensor bias, also after proton and gamma irradiation, with the exception of sensors irradiated by $10 \mathrm{kGy}$ gamma dose. The micro-discharge breakdown at $900 \mathrm{~V}$ is well above the maximum operating voltage $600 \mathrm{~V}$ and disappears after annealing and additional irradiation. The same instability at low radiation gamma doses has already been reported with the 
explanation that it is probably due to the large charge collected in the AC pad corners; after accumulating a higher dose, charges trapped in oxide layers act to reduce the electric field, enhancing the stability of the sensors [11]. The total leakage current increases $\sim 100$ times after gamma irradiation. It is most likely the result of the surface current increase. The surface damage is saturated after a relatively low dose and the total current does not change with increasing gamma dose. Unfortunately, the guard ring in the tested sensors does not have a probing pad and thus it is not possible to separate the leakage current of the inside of the bias ring from that of the outside of the bias ring.

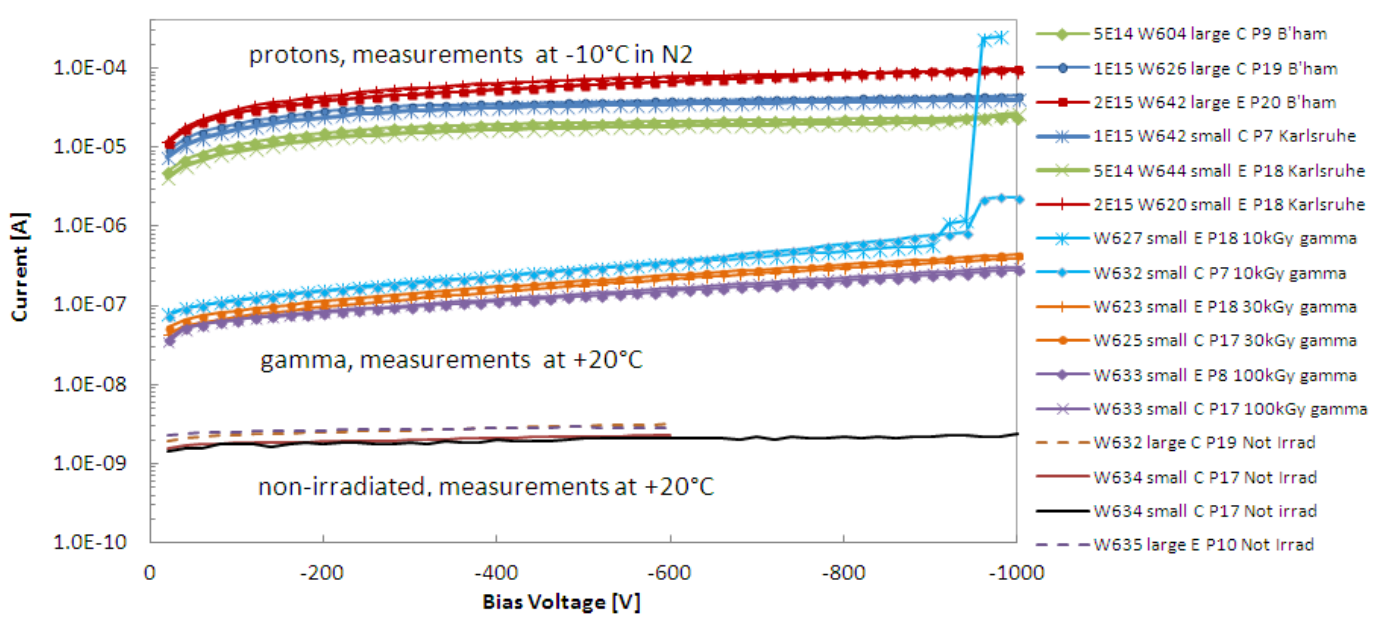

Figure 3: The leakage current vs. bias voltage for various sensors after proton and gamma irradiation compared with non-irradiated sensors.

\subsubsection{Bulk Capacitance and Full depletion voltage}

The bulk capacitance between the backplane and the bias ring as a function of bias voltage was measured with a HP 4284A precision LCR meter at a frequency of $1 \mathrm{kHz}$.

Examples of the inverse bulk capacitance squared $1 / C^{2}$ as a function of bias voltage for non-irradiated and gamma and proton irradiated sensors are presented in Fig. 4. Measurements of irradiated sensors were performed after controlled annealing at $60^{\circ} \mathrm{C}$ for 80 minutes. The $V_{F D}$ voltage was extracted from $\mathrm{CV}$ characteristics as the crossing of the extrapolated rising straight segment of the $1 / C^{2}(V)$ and the saturated value.

The $V_{F D}$ of non-irradiated ATLAS12A sensors estimated from measurements of 10 end-cap sensors is $358 \pm 15 \mathrm{~V}$. The full depletion voltage value allows for determination of the bulk resistivity of the silicon wafer, $\rho$ :

$$
\rho=d^{2} /\left(2 \varepsilon \mu V_{F D}\right) \approx 2.8 \pm 0.1 \mathrm{k} \Omega \mathrm{cm},
$$

where $d$ denotes the active thickness of sensor, $V_{F D}$ the full depletion voltage, $\varepsilon$ the dielectric constant of $S i\left(1.06 \times 10^{-12} \mathrm{~F} / \mathrm{cm}\right)$ and $\mu$ the hole mobility $\left(450 \mathrm{~cm}^{2} / V s\right)$. The active thickness of the wafer, $d=302 \mu \mathrm{m}$ was estimated from the saturated bulk capacitance value and active area of the sensors [12].

After proton irradiation the linear dependence in $1 / C^{2}$ curves is distorted and estimation of full depletion voltage is not clear. Nevertheless, Fig. 4 shows that the $V_{F D}$ increases with increasing proton fluence. Full depletion voltages, measured on 13 proton irradiated sensors, are 
in the ranges (580-800) V for $5 \times 10^{14}$ and (700-880) V for $1 \times 10^{15} \mathrm{n}_{\mathrm{eq}} / \mathrm{cm}^{2}$. Samples irradiated up to the highest proton fluence $2 \times 10^{15} \mathrm{n}_{\mathrm{eq}} / \mathrm{cm}^{2}$ did not reach full depletion even at $1000 \mathrm{~V}$.

$1 / C^{2}$ curves of the gamma irradiated samples do not exhibit any evolution and thus the $V_{F D}$ and effective doping concentration $\left(N_{\text {eff }}\right)$ is independent on gamma irradiation.

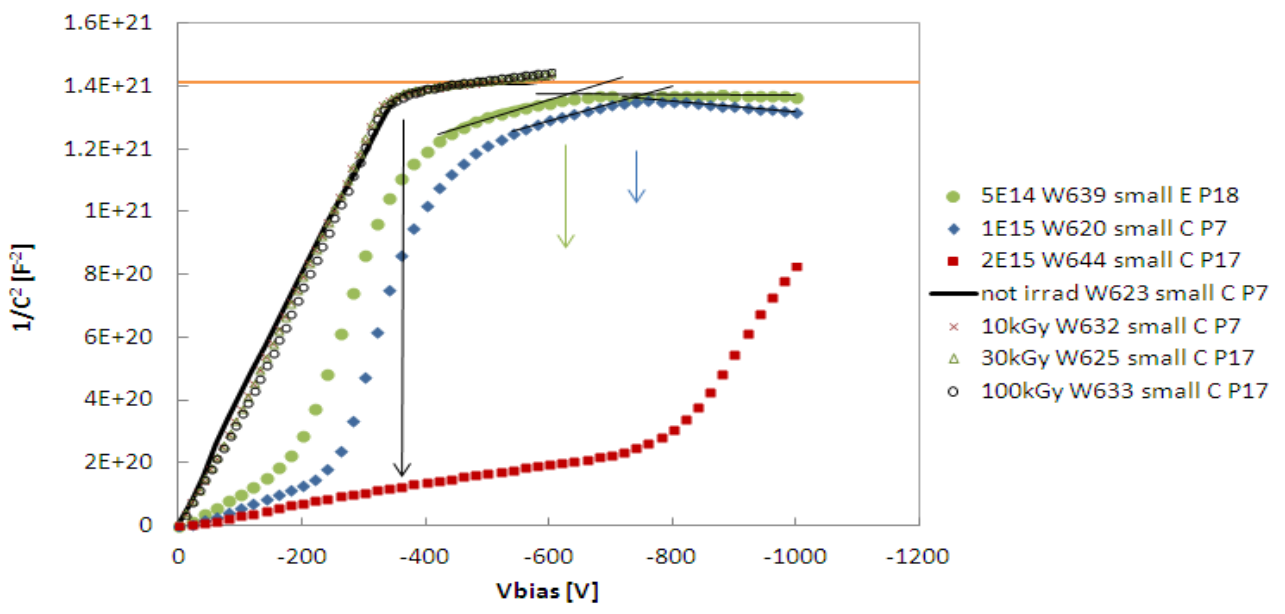

Figure 4: The bulk capacitance $\left(1 / C^{2}\right)$ of the EC miniature sensors of small pitch (active area = $0.686 \mathrm{~cm}^{2}$ ) as a function of bias voltage for un-irradiated sensors and for sensors irradiated by three radiation doses of gamma $(10,30,100 \mathrm{kGy})$ and three proton fluencies $\left(5 \times 10^{14}, 1 \times 10^{15}\right.$ and $\left.2 \times 10^{15} \mathrm{n}_{\mathrm{eq}} / \mathrm{cm}^{2}\right)$. The irradiated sensors are measured after annealing for 80 minutes at $60^{\circ} \mathrm{C}$.

\subsection{Surface damage}

\subsubsection{Inter-strip capacitance}

The inter-strip capacitance contributes to the input capacitance of the readout electronics and determines the noise level of the detector. Therefore, it should be as low as possible. The method of measuring inter-strip capacitance used in our study is shown in Fig. 5. The capacitance was measured between a central metal strip and its nearest neighbors as a function of frequency and bias voltage.

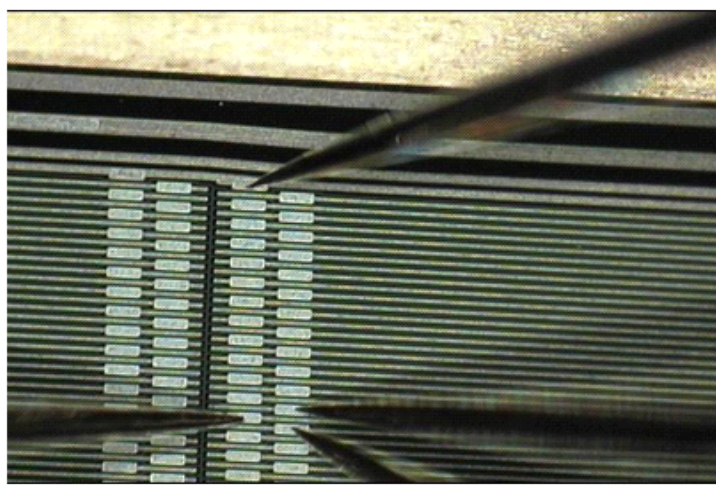

Figure 5: The inter-strip capacitance method in probe station: AC pads of metal strips are contacted with probes. The capacitance between the central strip and its neighbors is measured by an LCR meter.

The inter-strip capacitance of non-irradiated sensors measured at $100 \mathrm{kHz}$ test frequency as required by technical specifications is equal to 0.79 and $0.55 \mathrm{pF} / \mathrm{cm}$ for small and large pitch EC sensors, respectively, and 0.76 and $0.74 \mathrm{pF} / \mathrm{cm}$ for segment 1 and segment 2 of the skewed 
EC sensors, respectively. The difference of $\mathrm{C}_{\text {int }}$ for the two segments is due to theirs different average pitches.

The frequency dependence of the inter-strip capacitance for proton irradiated $\left(5 \times 10^{14}\right.$, $1 \times 10^{15}$ and $2 \times 10^{15} \mathrm{n}_{\mathrm{eq}} / \mathrm{cm}^{2}$ ) and for the non-irradiated sample is shown in Fig. 6a). The inter-strip capacitance measurements are dependent on the frequency of the AC signal. There is a resonance at $10 \mathrm{kHz}$ that increases with increasing proton fluence and also affects measurements of $\mathrm{C}_{\text {int }}$ at $100 \mathrm{kHz}$. Therefore, for irradiated sensors the $1 \mathrm{MHz}$ test frequency is more relevant than the $100 \mathrm{kHz}$ frequency indicated in specifications [10].
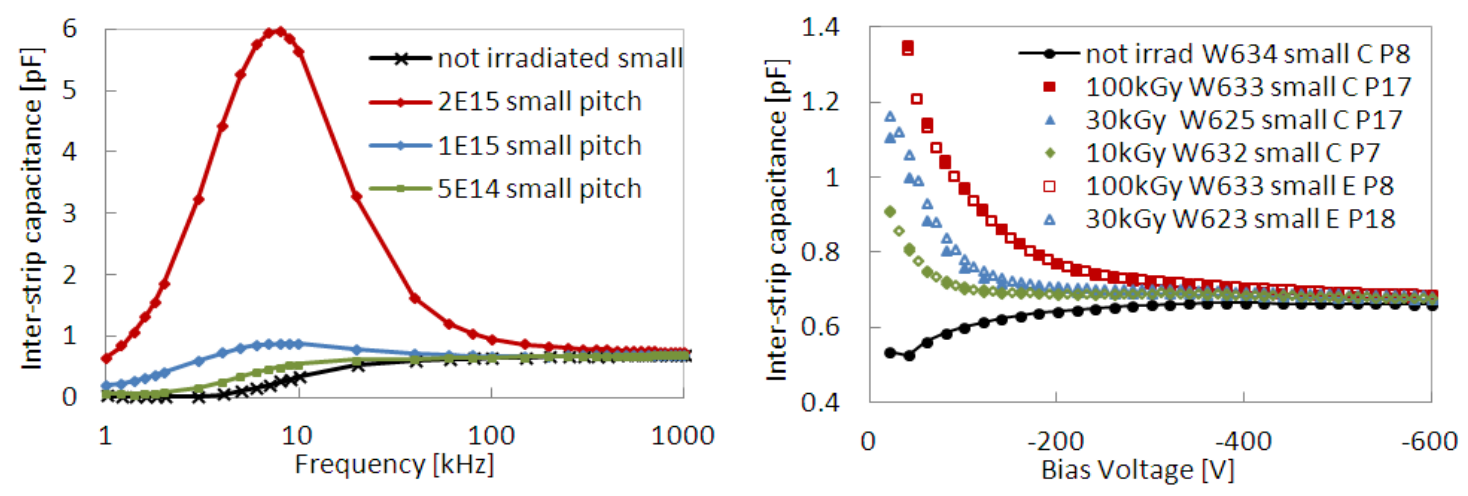

Figure 6: a) Frequency dependence of inter-strip capacitance after proton irradiation. b) Inter-strip capacitance as a function of bias voltage: for gamma irradiated ATLAS12A EC miniature sensors of small pitch.

The bias voltage dependence of $\mathrm{C}_{\text {int }}$ was measured as a function of proton fluence and gamma dose. The higher the radiation dose then the higher the value of the bias voltage needed for $\mathrm{C}_{\text {int }}$ to saturate to the pre-radiation value. Beyond $\mathrm{V}_{\mathrm{FD}}$ the $\mathrm{C}_{\mathrm{int}}$ becomes constant for both types of irradiation and for all tested doses. Fig. 6b) shows the results for gamma irradiation.

$\mathrm{C}_{\text {int }}$ is increased by $11 \%$ after proton irradiation and by $5 \%$ after gamma irradiation (Fig. 7 ).
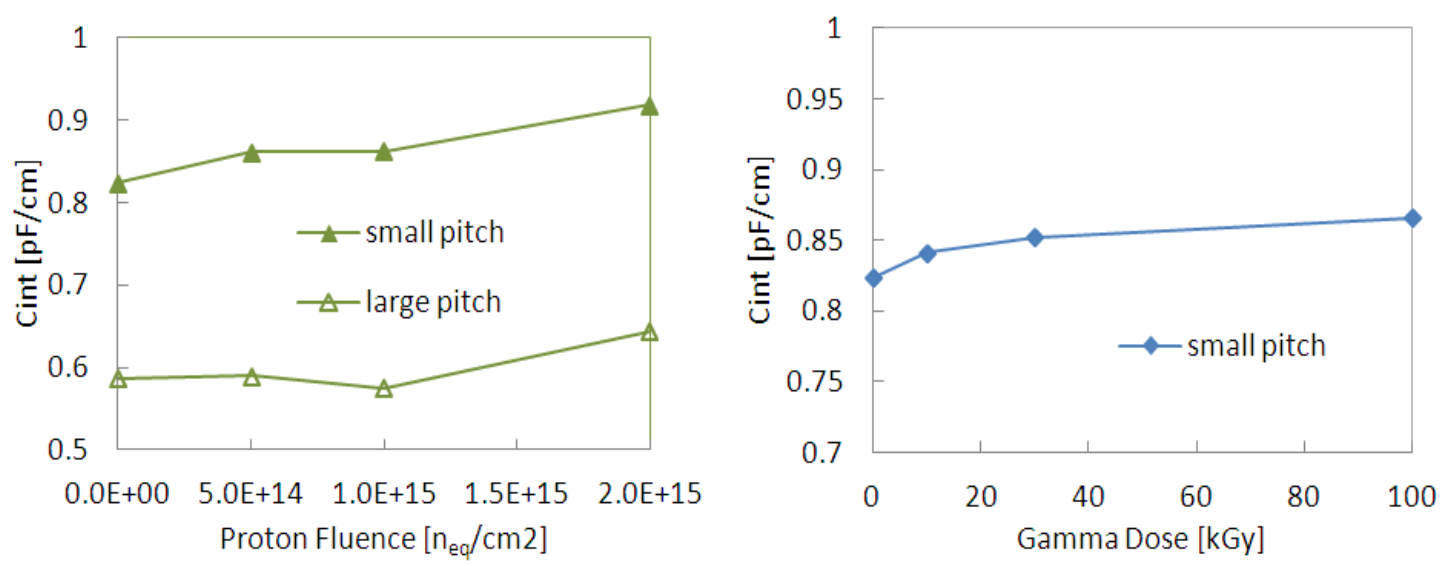

Figure 7: Inter-strip capacitance per $\mathrm{cm}$ as a function of proton fluence (left) and gamma dose (right) measured at $1 \mathrm{MHz}$ test frequency.

\subsubsection{Inter-strip resistance}

The isolation between neighboring strips is well demonstrated by the inter-strip resistance values $\left(\mathrm{R}_{\text {int }}\right)$. The inter-strip resistance measurements were performed by induced current method. 3 adjacent DC pads were contacted by 3 probes. On the outer strips a voltage $V_{\text {app }}$ was 
applied by SMU, the current $\mathrm{I}_{\text {int }}$ was measured on the central strip. The measurements were performed at $+20^{\circ} \mathrm{C}$ for non-irradiated sensors and at $-10^{\circ} \mathrm{C}$ for proton irradiated ones. Gamma irradiated sensors were measured both at $-10^{\circ}$ and $+20^{\circ} \mathrm{C}$ in order to determine the temperature dependence of $\mathrm{R}_{\text {int }}$. Nitrogen gas was flowing over the sensor for moisture control. The interstrip resistance was calculated by

$$
R_{\text {int }}=2 /\left(d I_{\text {int }} / d V_{\text {app }}\right) \text {. }
$$

The resulting IV characteristics for the proton irradiated sample, measured at different bias voltages, are shown in Fig. 8a).
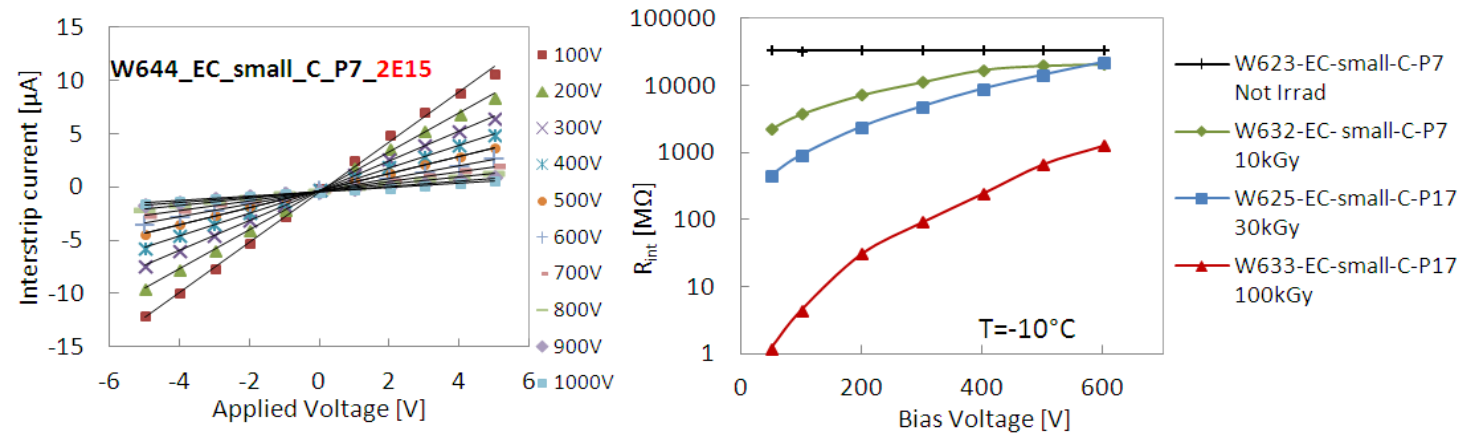

Figure 8: a) Inter-strip current as a function of applied voltage shown at different bias voltages. Sensor was irradiated by proton fluence $2 \times 10^{15} \mathrm{n}_{\mathrm{eq}} / \mathrm{cm}^{2}$. b) The inter-strip resistance as a function of bias voltage for non- irradiated and gamma irradiated sensors (gamma doses 10, 30, $100 \mathrm{kGy}$ ).

The inter-strip resistance for non-irradiated samples and samples irradiated by gamma irradiation to doses 10,30 and $100 \mathrm{kGy}$ are shown in Fig. 8b). The inter-strip resistance of non irradiated sensors is independent of bias voltage and its measured values are in the range 14$63 \mathrm{G} \Omega / \mathrm{cm}$, and thus exceed the minimum limit $10 \times \mathrm{R}_{\text {bias }} \sim 15 \mathrm{M} \Omega$ required in technical specification [10].

Inter-strip resistance after proton and gamma irradiation is reduced and strongly dependent on bias voltage. The dependence of $\mathrm{R}_{\mathrm{int}}$ on radiation dose is presented in Fig. 9a and 9b. For both type of irradiation $\mathrm{R}_{\text {int }}$ is decreasing with increasing radiation dose. The inter-strip resistance is seen to reduce from $\sim$ tens of $\mathrm{G} \Omega / \mathrm{cm}$ to hundreds of $\mathrm{M} \Omega / \mathrm{cm}$ as the gamma dose increases and to less than $10 \mathrm{M} \Omega / \mathrm{cm}$ as the proton fluence increases to $2 \times 10^{15}$ (measured at $-10^{\circ} \mathrm{C}$ ). As seen in
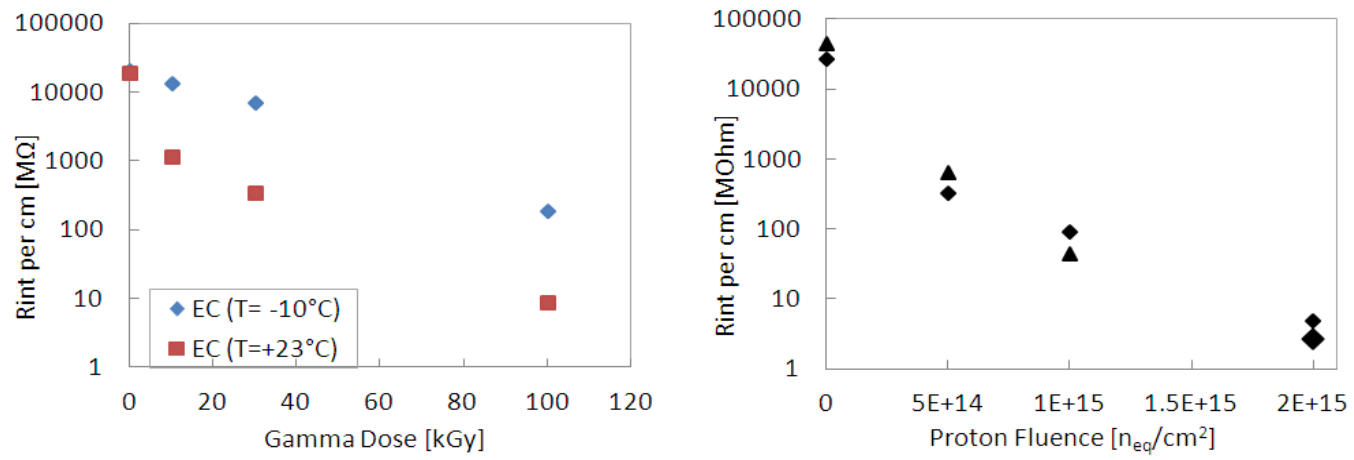

Figure 9: a) Inter-strip resistance as a function of gamma dose measured at $-10^{\circ}$ and $+20^{\circ} \mathrm{C}$ and b) proton fluence measured at $-10^{\circ} \mathrm{C}$. The bias voltage was $400 \mathrm{~V}$.

Fig. 9a) the values of $R_{\text {int }}$ measured at different temperature vary very strongly with temperature. The inter-strip resistance is related to the bulk resistivity and thus with the bulk leakage current, 
that changes with fluence and temperature [13]. By scaling the values of $\mathrm{R}_{\text {int }}$ measured at $-10^{\circ} \mathrm{C}$ to the operational temperature in ATLAS upgrade $\left(\sim-30^{\circ}\right.$ to $\left.-35^{\circ} \mathrm{C}\right)$ the $\mathrm{R}_{\text {int }}$ values are increased by factor of $\sim 8-12$.

\subsubsection{Punch-through protection}

AC coupled strip sensors are prone to large voltages between the readout metal strips and the implant strips. These large voltages can be caused by charge accumulation in the bulk due to beam splash accidents. A special protection structure, the punch-through protection structure, at the ends of the implant strips is used to keep the implant voltage low, by flowing the current though the structure at the voltages greater than the PTP onset voltage, thus avoiding breakdown.
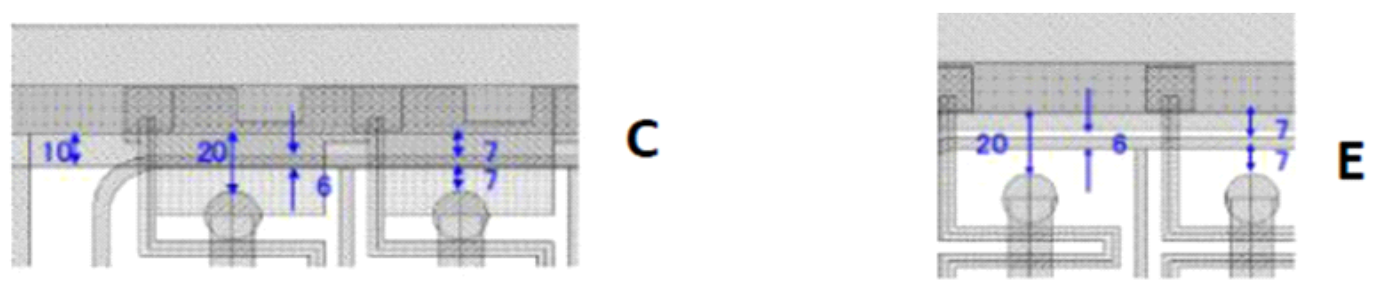

Figure 10: New PTP structure with full gate (C-type) and standard structure with no gate (E-type) [4].

The ATLAS 12A end-cap miniature sensors tested in this study have two types of PTP structures [3]; the PTP structure of C or E type. The E type is standard PTP structure with $20 \mu \mathrm{m}$ gap between the bias ring and the end of the implant $n^{+}$-strip and the $\mathrm{C}$ type is a new improved PTP structure with an extended PolySilicon electrode of the bias ring over the PTP gap; the fullgate structure (Fig. 10).

The effectiveness of the punch-through protection structure was tested using the DC method [14]: The test voltage $V_{\text {test }}$ was applied between the implant (DC pad) and the grounded bias rail. The effective resistance $R_{\text {eff }}$ was calculated from the resulting current $I_{\text {test }}$ and $V_{\text {test }}$ :

$$
R_{\text {eff }}=V_{\text {test }} / I_{\text {test }} \text {. }
$$

The effective resistance $R_{\text {eff }}$ consists of the punch-through resistance $R_{P T}$ in parallel to the polysilicon bias resistor $R_{\text {bias }}$. The punch-through voltage $\mathrm{V}_{\mathrm{PT}}$ is then defined to be the test voltage at which $R_{P T}=R_{\text {bias }}$, i.e. for $R_{\text {eff }}=R_{\text {bias }} / 2$.

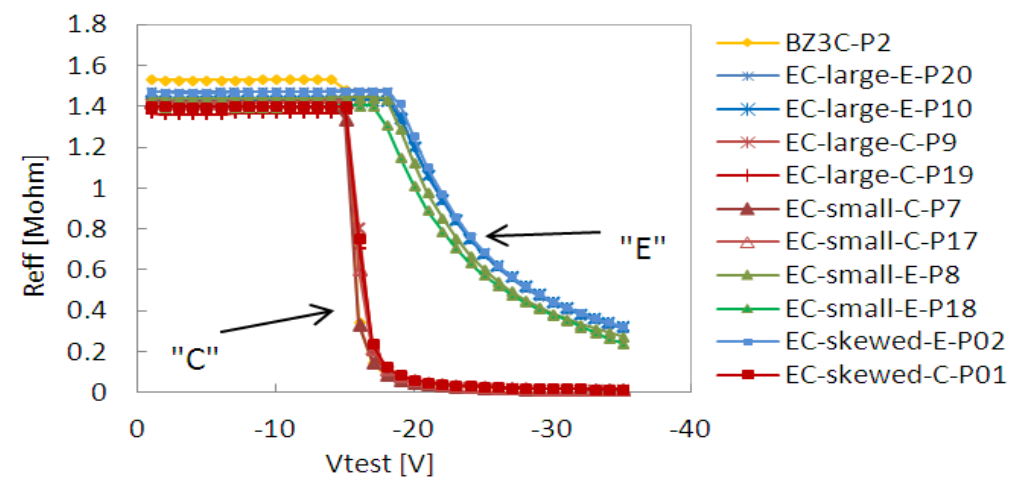

Figure 11: Effective resistance Reff as a function of applied test voltage Vtest for non-irradiated sensors with C and E type of PTP structure taken at bias voltage 400V.

Gamma and proton irradiated sensors have lower inter-strip resistance and inter-strip currents as well as current from the bulk influence the PTP measurements. To exclude inter-strip 
effects the voltage $V_{\text {test }}$ was applied also on two neighbors at the same time, IV was performed on the central strip only.

A well functioning PTP structure demands low $V_{P T}$, a quick drop of $R_{\text {eff }}$ after $V_{P T}$ and as low as possible resistance at large voltages.

The effective resistance as a function of the test voltage for all tested non-irradiated sensors is shown in Fig. 11 while irradiated sensors are shown in Fig. 12. The C type structure shows better performance than the $\mathrm{E}$ type: lower punch-through, $V_{P T}(\mathrm{C}$-type $)=15.9 \pm 0.2 \mathrm{~V}, V_{P T}$ $($ E-type $)=24.0 \pm 0.7 \mathrm{~V}$, lower effective resistance, $R_{\text {eff }}\left(\mathrm{C}\right.$-type) $10 \mathrm{k} \Omega, R_{\text {eff }}($ E-type $) 300 \mathrm{k} \Omega$ and very steep drop of $R_{\text {eff }}$ around $V_{P T}$.
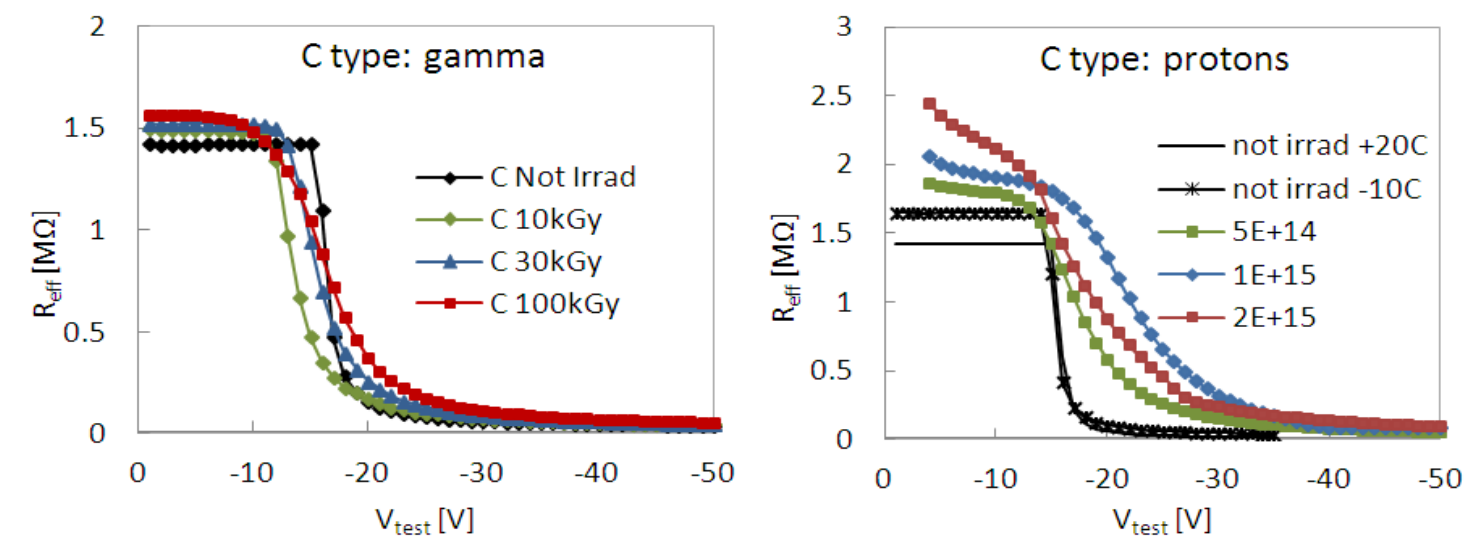

Figure 12: Effective resistance Reff as a function of applied test voltage Vtest for sensors irradiated by gammas (left) and protons (right) for gated PTP structure (type C). As an example Reff of non-irradiated sensors is also included for comparison.

It can be seen in the left plot of Fig. 12 that gamma irradiation does not change the effectiveness of $\mathrm{C}$ type of PTP structure. The punch-through voltage stays in the range 14-17 V and the effective resistance at $50 \mathrm{~V}$ is $\sim 50 \mathrm{k} \Omega$. Protons have a stronger influence on $R_{\text {eff }}$, as seen in right plot of Fig. 12. The punch-through voltage increases to $24 \mathrm{~V}$ after proton irradiation and the decrease of $R_{e f f}$ around $V_{P T}$ is not so sharp. The effective resistance is increasing with fluence from $20 \mathrm{k} \Omega$ before irradiation to $100 \mathrm{k} \Omega$ after highest fluence $2 \times 10^{15} \mathrm{n}_{\mathrm{eq}} / \mathrm{cm}^{2}$.

\section{Summary and Conclusions}

The electrical characteristics of the end-cap miniature sensors, ATLAS12A, before and after both proton and gamma irradiation were evaluated in Prague. All sensors have a high micro-discharge breakdown voltage $>1000 \mathrm{~V}$ (not irradiated) and $>900 \mathrm{~V}$ (irradiated) which is well above the maximum operational voltage. It shows that different geometries of end-cap sensors do not influence their stability. The full depletion voltage extracted from CV characteristics is $\sim 360 \mathrm{~V}$ for non-irradiated sensors, which corresponds with bulk resistivity $\approx 3 \mathrm{k} \Omega \mathrm{cm}$. The full depletion voltage does not change with gamma irradiation but rises with proton irradiation. At the highest tested fluence $2 \times 10^{15} \mathrm{n}_{\mathrm{eq}} / \mathrm{cm}^{2}$, the $\mathrm{V}_{\mathrm{FD}}$ is much greater than $1000 \mathrm{~V}$. It is expected that the sensors will operate partially depleted in the upgraded ATLAS at HL-LHC. The inter-strip capacitance is increased by $11 \%$ after proton, and by $5 \%$ after gamma irradiation, which is still acceptable with regard to the low noise of these sensors. The inter-strip resistance degrades strongly with proton and gamma irradiation. However for the highest tested proton fluence, $2 \times 10^{15} \mathrm{n}_{\mathrm{eq}} / \mathrm{cm}^{2}$, and gamma dose, $100 \mathrm{kGy}$, at operating temperature $-30^{\circ} \mathrm{C}$ its 
value is much larger than the typical amplifier's input impedance of $1 \mathrm{k} \Omega$ and thus the strip isolation is sufficient. Radiation damage caused by protons reduces the effectiveness of the PTP structure. However the new PTP structure with gate (C-type) shows significantly better protection than the standard E-type without gate. The gate PTP structure functions well even at the highest proton fluence.

\section{Acknowledgements}

We acknowledge the support by the European Commission within the Framework Program 7 Capacities, Grant Agreement 262025 and by the Ministry of Education, Youth and Sports of the Czech Republic under the project Nr. 7E12050, the German Federal Ministry of Education and Research, and the Helmholtz Association, the Japan Society for Promoting Science KAKENHI A (Grant number 20244038) and KAKENHI C (Grant number 20540291), the Japan MEXT KAHENHI for Research on Priority Area (Grant number 20025007) and for Scientific Research on Innovative Areas (Grant number 23104002), the Slovenian Research Agency, the Spanish National Program for Particle Physics (under Grants FPA2009-13234-C04-01 and FPA2012-39055-C02-01), the financial support of the State Secretariat for Education, Research, and Innovation, the Swiss National Science Foundation and the Canton of Geneva, Switzerland, the UK Science and Technology Facilities Council (under Grant PP/E006701/1), and the United States Department of Energy, Grant DE-FG02-04ER41286.

\section{References}

[1] ATLAS Collaboration, Letter of Intent Phase II Upgrade, CERN-LHCC-2012-022 LHCC-I-023.

[2] Y. Unno et al., Nucl. Instr. and Meth. A636 (2011) S24.

[3] Y. Unno et al., Nucl. Instr. and Meth. A765 (2014) 80.

[4] Hamamatsu Photonics K.K. http://www.hamamatsu.com .

[5] P. Dervan et al., The Birmingham Irradiation Facility, Nucl. Instr. and Meth. A730 (2013) 101.

[6] Karlsruhe Institute of Technology, Irradiation center, Hermann-von-Helmholtz-Platz 1, 76344 Eggenstein-Leopoldshafen, Germany.

[7] Solid State Gamma-ray irradiation facility, Brookhaven National Laboratory, Instrmental Division, Bldg. 535B, 20 N. Technology Street, Upton, NY 11973.

[8] J. Bohm et al., Nucl. Instr. and Meth. A636 (2011) S104.

[9] J. Bohm et al., 8th "Trento" Workshop on Advanced Silicon Radiation Detectors (3D and p-type) http://indico.cern.ch/event/226647/session/3/contribution/25/material/slides/1.pdf .

[10] ATLAS Upgrade Sensor Collaboration, private communication.

[11] K. Hara et al., Nucl. Instr. and Meth. A699 (2013) 107.

[12] The active thickness of ATLAS12A sensors $d=302 \mu m$ was estimated from bulk capacity measurements provided by different laboratories on large area sensors, barrel and end-cap miniature sensors.

[13] Y. Unno et al., Nucl. Instr. and Meth. A731 (2013) 183.

[14] S. Lindgren et al., Nucl. Instr. and Meth. A636 (2011) S111. 